\title{
Perception of Head Motion Effect on Emotional Facial Expression in Virtual Reality
}

\author{
Qiongdan $\mathrm{CaO}^{1}$ \\ University of Portsmouth \\ Hui Yu2* \\ University of Portsmouth \\ Charles Nduka ${ }^{3}$ \\ Emteq Ltd, Brighton
}
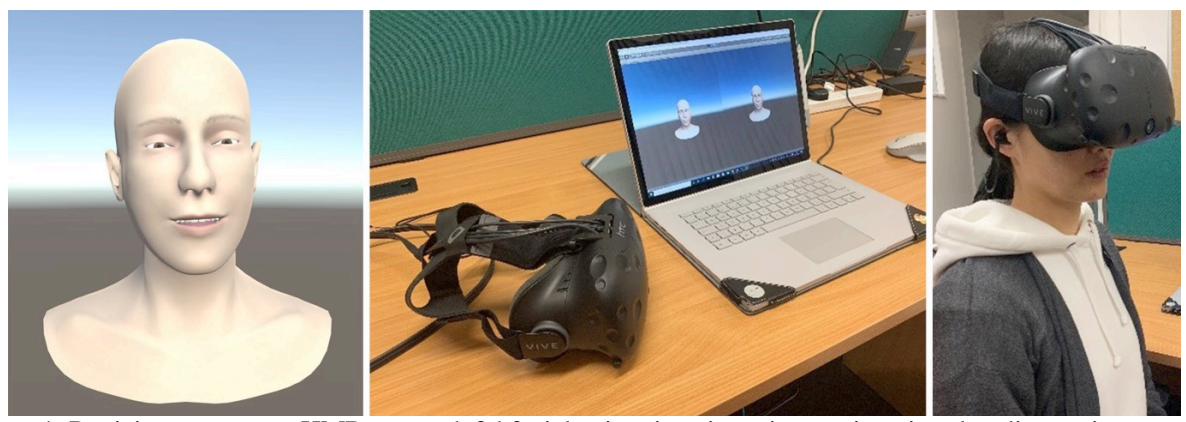

Figure 1: Participants wear an HMD to watch $3 \mathrm{~d}$ facial animations in an immersive virtual reality environment. An example of facial expressions in VR (left); experiment environment (middle); a participant wearing an HMD (right).

\begin{abstract}
In this paper, we present a study investigating the impact of head motion on realism, perceived emotion intensity and affinity for facial expressions to enhance the realism of interactive virtual characters. We designed an experiment to measure the impact through a combination of methods including subject behavioural data rating designed facial animations in Virtual Reality (VR) and questionnaire ratings. The result shows head motion has positive impact on facial expressions that enhance realism, perceived emotion intensity and affinity for virtual characters in one emotion situation.
\end{abstract}

Keywords: Realism; Head motion; Facial expression; Emotion; Virtual reality;

\section{INTRODUCTION}

Virtual Reality (VR) becomes an important platform for games, education, and therapy. Virtual character plays a crucial role in VR because users will feel engaged with interactive virtual characters. It is thus important to identify features that interactive virtual characters may possess. Current research has investigated factors such as rendering style [4], body ownership [7], and personality [3] for increasing the appearance of virtual characters which contribute to character design.

Facial expression displays non-verbal communication and conveys emotion information in our daily life interactions. Head motion combined with facial expression shows complex relationship between facial expressions. Some pilot research has

'email: qiongdan.cao@myport.ac.uk

2"email: hui.yu@port.ac.uk (correspondence)

semail: charles@emteq.net demonstrated that head motion can convey emotion information by itself in some specific situations [6]. However, there are still some open questions about head motion regarding the realism of facial expression. 1) whether the realism of the virtual character would be influenced by adding head motion to dynamic facial expressions 2) whether the perceived emotion intensity of a dynamic facial expression of a virtual character would be increased by adding head motions. 3) whether the realism of facial expression would be improved in a positive way that can be measured using affinity according to Uncanny Valley [5]. In this paper, we present a study that focuses on exploring the impact of head motion on three aspects: realism, perceived emotion intensity and affinity of facial expressions for virtual characters in VR using perception method [2] in order to get ideas for better character design.

\section{Experiment Design}

We have designed a behavioral experiment by using participants to rate the designed facial animation which are created based on a group of 3D facial blend shape models. These facial animations are integrated into a fully immersive VR environment during the experiment. We also designed a questionnaire to collect rating data from randomized participants. The questions were rated on a $10-$ point Likert scale, rating from $1-$ Not at all to $10-$ Exactly.

\subsection{Experiment Set Up and Stimuli}

The experiment environment was set in a quiet research lab at the University of Portsmouth. Participants needed to wear a Head Mounted Display (HMD) during the experiment and watch facial animations in a fully immersive VR environment. In this experiment, the facial animations were locked in one view and only used for the observation purpose which wasn't interactable.

We created 12 different animations according to real life videos for the stimuli in VR [1]. Firstly, we investigated real-life captured videos online that might contain both head motion and facial expression at the same time. We have collected a set of videos that recorded different people's reaction to being called beautiful. These videos contain not only facial expressions and head motions but also emotion responses, which serve well for our stimuli requirements. We selected 6 video clips as the references for 
creating dynamic 3D facial animations in VR for our experiment. It was a challenge to collect videos online with different emotions such as sad and angry that meet our animation requirements. Most of the emotion response in the collected video is happy and sometimes combines with a little bit surprise and shy. Secondly, we analyzed the videos and separated the motions into parts - facial expression, head motion and body motion. We removed body motion on purpose to allow to focus on head motion and facial expression. In this experiment, we excluded other emotions such as surprise and shy and only remained a single happiness expression, which allow to recreate these facial expressions in the 3D world according to Facial Action Coding System (FACS). However, we kept head motions the same as the original videos. Finally, we created the 3D facial animations by using blend shapes in VR. We matched the head motion in the video clips to the corresponding 3D character. For example, in the reference video, the actor smiled and nodded for 3 seconds, so the 3D model also smiled and nodded for 3 seconds in our experiment.

We classified the animations into two groups. One is the facial animations with head motions (HM) and another one is the facial animations without head motions (NHM). Each group has 6 different animations. The facial expressions are kept the same in both groups. The only difference between these two groups is that one has head motion and the other one doesn't have. Figure 2 shows screen capture examples of the animation.

\subsection{Experiment Procedure}

In total, 24 participants (10 males, 14 females; aged 18 above) volunteered to do the experiment. These participants included both students and staff at the University of Portsmouth. All of them have a normal vision or have a normal vision that can be fixed by wearing a pair of glasses. The research aim and experiment procedure were explained to the participants before starting the actual experiment. Participants were given time to read the paper instructions and were given oral explanation before starting the experiment. Then, participants were required to wear the HMD to test if the HMD worked or not. Finally, participants watched the animations using HMD and filled in the questionnaire by rating the realism, emotion, affinity for each animation. The duration of the animation in total was 3.5 minutes and each single animation was played twice.

\section{Result}

We firstly calculated the mean value of the ratings from the same animation group for each participant. Then we used a paired samples T-test to analyze the difference of three dependent variables between different animation groups (HM vs NHM). Significant differences on realism, perceived emotion intensity, and affinity were found between HM and NHM. The statistical data show as below:

Realism: the result of question1 "I found the character was realistic" shows that HM $(M=7.05, S D=1.29)$ was rated higher than NHM $(M=6.34, S D=1.37)$ with $t(23)=2.76, p=0.011$.

Perceived Emotion Intensity: the result of question2 "I found the character was happy" shows that HM $(M=7.39, S D=1.28)$ was rated higher than NHM $(M=6.21, S D=1.38)$ with $t(23)=5.01, p$ $<0.001$.

Affinity: the result of question3 "I found I wanted to interact (communicate) with the virtual character after watching the animation" shows that HM $(M=6.65, S D=1.58)$ was rated higher than NHM $(M=5.49, S D=1.43)$ with $t(23)=6.0, p<0.001$.

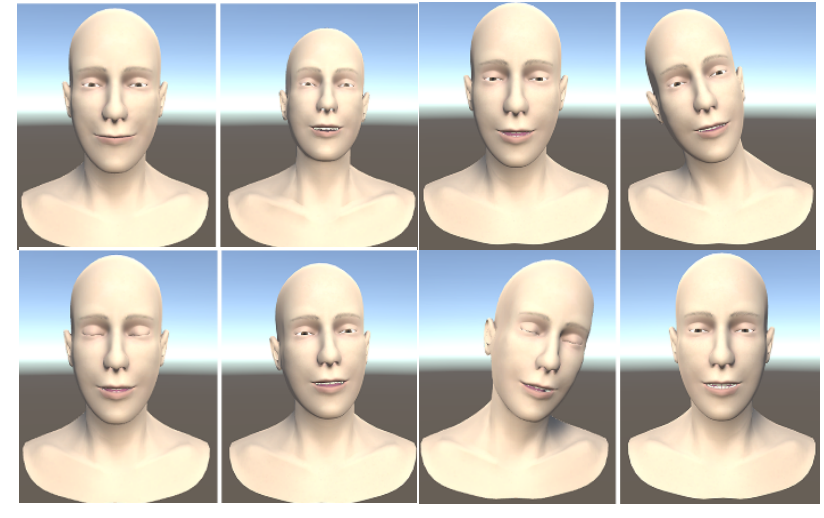

Figure 2: Screen capture examples of the animation.

\section{Conclusion}

Our study tested the role of head motion on emotion presentation along with facial expressions in VR. The experiment showed that the realism of facial expressions was enhanced by adding head motions. Head motion assisted facial expressions conveying emotions to participants and participants were more willing to interact with the virtual character with head motions. The statistics data indicates that head motion has an impact on facial expressions in VR in three different aspects.

However, we only explored a single emotion situation in this experiment. There are lots of emotion situations in real life and there may be different results according to different emotions in the same experiment. Therefore, we will design more comprehensive experiment for different emotion situations in future.

\section{References}

[1] G. Held, I Think You're Beautiful! I Social Experiment. Retrieved from: https://www.youtube.com/watch? $=\mathrm{gW} 8 \mathrm{wV} \_q a a Q c, \quad J u l y$ 2018.

[2] H. Yu, O. G.B. Garrod, P. G. Schyns. "Perception-driven facial expression synthesis". Computers \& Graphics, ELSEVIER, Vol 36, No.3,pp:152-162, 2012.

[3] K. Zibrek, E. Kokkinara, R. McDonnell. "The Effect of Realistic Appearance of Virtual Characters in Immersive Environments - Does the Character's Personality Play a Role?" IEEE Transactions on Visualization and Computer Graphics, Vol 24, No 4, April 2018.

[4] K. Zibrek, E. Kokkinara, and R. McDonnell. "Don't stand so close to me: investigating the effect of control on the appeal of virtual humans using immersion and a proximity - based behavioral task". In Proceedings of the ACM Symposium on Applied Perception, p. 3. ACM, 2017.

[5] M. Mori. The uncanny valley. Energy, 7(4): 33-35, 1970.

[6] S. R. Livingstone, and C. PalmerS. "Head movements encode emotions during speech and song". Emotion 16:365, 2016, doi: 10.1037/emo0000106

[7] T. Waltemate, D. Gall, D. Roth, M. Botsch and M.E. Latoschik. "The Impact of Avatar Personalization and Immersion on Virtual Body Ownership, Presence, and Emotional Response". IEEE Transactions on Visualization and Computer Graphics, 24 (4): 1643 - 1652, April 2018. doi: 10.1109/TVCG.2018.2794629. 